\title{
A Survey on Medication Reviews in Older Patients: Substantial Variation in Daily Practice
}

Kim PGM Hurkens ${ }^{1 *}$, Carlota Mestres-Gonzalvo ${ }^{2}$, Hugo AJM de Wit ${ }^{2}$, P. Hugo M van der Kuy ${ }^{2}$, Rob Janknegt ${ }^{2}$, FransVerhey ${ }^{3}$, Jos MGA Schols ${ }^{4}$, Leo ML Stolk ${ }^{5}$, Coen DA Stehouwer ${ }^{1,6}$ and Wubbo Mulder ${ }^{1}$

${ }^{1}$ Department of Internal Medicine, Maastricht University Medical Centre, The Netherlands ${ }^{2}$ Department of Clinical Pharmacology, Orbis Medisch Centrum Sittard, The Netherlands

${ }^{3}$ Alzheimer Centrum Limburg, School of Mental Health and Neuroscience, Maastricht University Medical Centre, The Netherlands

${ }^{4}$ Department of Family Medicine, Maastricht University Medical Centre, The Netherlands

${ }^{5}$ Department of Clinical Pharmacology, Maastricht University Medical Centre, The Netherlands

${ }^{6}$ Cardiovasculair Research Institute Maastricht (CARIM), The Netherlands

\begin{abstract}
Objective: To assess the methods and frequency by which medication reviews are performed by general practitioners and nursing home physicians by means of a survey.

Methods: 134 nursing home physicians and general practitioners working in the southern part of the Netherlands, the province of Limburg were asked to fill in a digital questionnaire. Non response was followed by second emailing and a questionnaire on paper by regular post. The questionnaire was developed by an expert panel, consisting of two hospital pharmacists, an internist, a nursing home physician and a neuropsychiatrist.

Results: There was substantial inconsistency in the frequency of performing medication reviews, ranging from monthly (in $40 \%$ ) by the nursing home physicians to four times a year (in $50 \%$ ) by the general practitioners. Time spent on one review also varied significantly between groups, namely 10 minutes for a nursing home physician and 20 minutes for general practitioners. Meetings between the physician and pharmacist took place regularly (91\%), but these were not organised for medication reviewing of individual patients. When medication was changed by another doctor, $47 \%$ of nursing home physicians and $44 \%$ of the general practitioners were informed often, whereas $40 \%$ and $50 \%$ respectively were only informed sometimes, and $13 \%$ of nursing home physicians and $6 \%$ of general practitioners never received any notice. $59 \%$ of the nursing home physicians and $89 \%$ of general practitioners considered workload to be a limiting factor in performing reviews.
\end{abstract}

Conclusions: This survey shows great inconsistency in the way medication reviews are done. To achieve a high standard, we may have to reconsider the way medication reviews are done.

Keywords: Medication review; Older people; Nursing home; General practice

\section{Introduction}

The rising incidence of multi-morbidity among older people makes polypharmacy a global challenge. The prevalence of multi-morbidity among those 65 years and older has been estimated at $60 \%$ worldwide $[1,2]$. More than $90 \%$ of persons aged 65 years or older use at least one drug per week. Polypharmacy defined as $\geq 5$ medicines per person was present in $19 \%$ of men and $23 \%$ of women aged 65 or older in an ambulatory setting [3]. In 2011 there were 2.6 million inhabitants aged 65 or older in the Netherlands [4]. Assuming similar prevalence to the USA, this would mean that polypharmacy is present in approximately 540.000 of older Dutch patients. In the UK, there are approximately 400.000 older residents living in care homes, which comprise $4 \%$ of the UK population aged 65 and over. These residents had a prescription for 7.2 medicines on average $[5,6]$. Polypharmacy increases the risk on adverse drug reactions, such as cognitive loss, delirium, falls, fractures, urine-incontinence, renal function loss, haemorrhage, unnecessary hospital- or nursing home admissions, and even death [7-11]. A Dutch study showed that, of all unplanned hospital admissions, 5.6\% were medication-related [10]. Almost half (46.5\%) of these admissions were considered to be potentially preventable. The mean age of the patients with a potentially preventable hospital admission was significantly higher (68 years) than the age of the patients with an unplanned admission (60 years). Polypharmacy was one of the main risk factors [10].

Therefore, adequate medication management is an important cornerstone in the care for older people.
In the Netherlands, daily and long-term medical care for older patients is provided by general practitioners when the patient lives independently in the community or in a residential home. When morbidity and disability are so severe that independent living is no longer possible, they are admitted to a nursing home, where nursing home physicians are responsible for the medical care [12]. In 2012 there were about 365 nursing homes, with approximately 65,000 beds.

According to the Dutch survey of the Health Care Inspectorate: medication safety for vulnerable groups in long-term care and at home is insufficient', a yearly medication review should be performed for every older person by a physician, pharmacist, nurse, and if possible with the patient him/herself. For nursing home patients the review should be performed twice a year [13]. However, performing medication reviews this way is very time-consuming and demands frequent meetings between physician, pharmacist, nursing

*Corresponding author: Kim PGM Hurkens, Department of Internal Medicine, Maastricht University Medical Centre, PO box 5800, Maastricht, The Netherlands Tel: +31 433877005; Fax: +31 433875006; E-mail: k.hurkens@mumc.nl

Received July 10, 2013; Accepted September 30, 2013; Published October 03, 2013

Citation: Hurkens KPGM, Mestres Gonzalvo C, de Wit HAJM, van der Kuy PHM, Janknegt R, et al. (2013) A Survey on Medication Reviews in Older Patients: Substantial Variation in Daily Practice. J Gerontol Geriat Res 2: 133 doi:10.4172/2167-7182.1000133

Copyright: $\odot 2013$ Hurkens KPGM, et al. This is an open-access article distributed under the terms of the Creative Commons Attribution License, which permits unrestricted use, distribution, and reproduction in any medium, provided the original author and source are credited. 
staff and the patient. Therefore, it is presumed that these requirements will be hard to meet in daily practice.

Moreover, despite this general advice, it is largely unknown how (and whether) this is practised at present. The aim of this study was to examine the methods and frequency by which medication reviews are performed by general practitioners and nursing home physicians, using a web-based questionnaire.

\section{Methods}

\section{Subjects}

Nursing home physicians and general practitioners working within the area of postal codes 6211 to 6325 , located in the southern part of the province of Limburg, the Netherlands.

\section{Questionnaire}

A digital questionnaire (supplement) was sent to all subjects. Non response was followed by a second emailing. Finally, the professionals were approached by means of a letter and questionnaire on paper by regular post. The questionnaire was developed by an expert panel, consisting of two hospital pharmacists, an internist, a nursing home physician and a neuropsychiatrist. The survey involved questions regarding a number of characteristics of the physicians and the concrete process of medication reviewing, i.e. the accessibility of information in relation to medication reviews, which care providers are included in the review, the process of prescribing and distribution of medication, time management, possible consequences of the medication review and the actual workability of a medication review. For usability the initial questionnaire was scrutinized by peers after whom questions were deleted or refined. Data were analysed using the software IBM SPSS statistics 21 . Values are presented as median values and interquartile range because of non-normal distribution. Correlation between frequency of reviewing and time spent on one medication review was calculated with the Pearson's correlation coefficient.

\section{Results}

\section{Characteristics of subjects}

Of the 134 subjects who received a questionnaire, 63 (47\%) responded. Of these 63 subjects, eighteen (29\%) were general practitioners and $45(71 \%)$ nursing home physicians. The median (interquartile range) level of experience of nursing home physicians was $14(8-22)$ years while the general practitioners had 13 (9-21) years' experience (Table 1). The median number of old people under the care of nursing home physicians was 60 (40-80). For general practitioners this was 50 (20-70). All nursing home physicians worked in a team. Three out of 18 general practitioners had a solo practice.

\section{Process of medication reviewing}

All physicians reported that they performed medication reviews. The frequency with which they performed reviews ranged from daily (8\%) to once a year (5\%). Most of the reviews were said to be done four times a year (50\%) by the general practitioners and monthly (40\%) by the nursing home physicians. The frequency of reviewing did not seem to influence the time spent on one review. The median number of patients reviewed per year was reported to be 85 (50-200) for the nursing home physicians and 30 (20-50) for the general practitioners. The medication reviews were mostly performed by the physician (84\%) alone. In $91 \%$ of cases, regular meetings between pharmacist and physician took place, but the frequency varied from weekly to yearly. Most of the nursing home physicians said to have monthly meetings
(63\%) whereas most of the general practitioners had a meeting with the pharmacist four times a year (60\%). These meetings were organised as pharmacotherapy audit meetings (FTOs) in 69\% in both groups. In $14 \%$ of nursing home physicians and $31 \%$ of general practitioners, these meetings were arranged as medication review meetings and $7 \%$ of nursing home physicians did both. $10 \%$ of nursing home physicians also reported had other forms of consultations with the pharmacist such as by telephone. In the nursing homes, $40 \%$ of the patients are alleged to have been participating in the review whereas in the general practitioners practices $28 \%$ of the patients participated in the review. The physicians considered the doctor $(86 \%)$ or the pharmacist $(90 \%)$ the best professional to carry out the medication review, preferably together.

Median time spent on a review reported for one patient was 10 minutes (5-14) for nursing home physicians and 20 minutes (13-30) for general practitioners. The median number of interventions proposed after a review was 2 (1-2) and was the same in both groups. The median number of executed interventions was said to be 1 (1-2). The most important interventions mentioned were discontinuing medication and altering dosages. Seventy-three per cent of the physicians only reviewed their own patients. Fifty-nine percent of nursing home physicians and $89 \%$ of general practitioners considered workload to be a limiting factor in performing reviews.

With regard to what type of information was used when reviewing, $88 \%$ said to use laboratory values and $75 \%$ said to use patient characteristics to decide whether to start, stop or alter medication.

In case medication was changed by another doctor, for instance by a hospital specialist, $47 \%$ of the nursing home physicians and $44 \%$ the general practitioners reported to be informed often and $40 \%$ respectively $50 \%$ were informed sometimes. On the other hand $13 \%$ of nursing home physicians and $6 \%$ of general practitioners said they never received any notice of colleagues regarding medication alterations. Of nursing home physicians, $84 \%$ reported always to notify the pharmacy when altering drugs compared to $65 \%$ of general practitioners. No notification was sent in $9 \%$ and 24 respectively and

\begin{tabular}{|l|c|c|c|}
\hline & Total & NHP & GP \\
\hline Experience (years) $^{*}$ & $14(9-22)$ & $14(8-22)$ & $13(9-21)$ \\
\hline Number of patients* & $60(33-80)$ & $60(40-80)$ & $50(20-70)$ \\
\hline Frequency of review $^{\#}$ & & & \\
Daily & $5(8)$ & $2(4)$ & $3(17)$ \\
Weekly & $7(11)$ & $4(9)$ & $3(17)$ \\
Monthly & $20(32)$ & $18(40)$ & $2(11)$ \\
Quarterly & $20(32)$ & $11(24)$ & $9(50)$ \\
Semi yearly & $8(13)$ & $7(16)$ & $1(6)$ \\
Yearly & $3(5)$ & $3(7)$ & $0(0)$ \\
\hline Number of patients reviewed per year* & $60(30-120)$ & $85(50-200)$ & $30(20-50)$ \\
\hline Time investment per review (min)* & $10(5-21)$ & $10(5-14)$ & $20(13-30)$ \\
\hline Number of proposed adjustments* & $2(1-2)$ & $2(1-2)$ & $2(1-2)$ \\
\hline Number of carried out adjustments* & $1(1-2)$ & $1(1-2)$ & $1(1)$ \\
\hline Workload restriction ${ }^{*}$ & & & \\
Yes & $42(67)$ & $26(59)$ & $16(89)$ \\
\hline No & $20(32)$ & $18(41)$ & $2(11)$ \\
\hline Feedback from other doctors ${ }^{*}$ & & & \\
Often & $29(46)$ & $21(47)$ & $8(44)$ \\
Sometimes & $27(43)$ & $18(40)$ & $9(50)$ \\
\hline Never & $7(11)$ & $6(13)$ & $1(6)$ \\
\hline
\end{tabular}

*Indicating median (interquartile range)

\#Indicating absolute numbers (percentages), NHP = nursing home physicians, GP = general practitioners

Table 1: Results of medication review by nursing home physicians and genera practitioners. 
in $7 \%$ of nursing home physicians and $12 \%$ of general practitioners a notification was sent sometimes.

A medication distribution protocol is a document in which the responsibility and competences with regard to the distribution and administration of drugs is determined. In $96 \%$ of nursing homes and $61 \%$ of general practices such a protocol seems to be available. In the nursing homes, $62 \%$ of drugs was said to be administered by the nursing staff while $38 \%$ was self-administered. For the older general practice population, in $62 \%$ home care nurses administered the drugs and in $39 \%$ it involved self-administered medication. $76 \%$ of all nursing home physicians and $100 \%$ of general practitioners stated to use an electronic medication surveillance program.

In $84 \%$ of nursing homes and in $50 \%$ of general practices there was said to be a drug formulary available, which was lacking in 16 and $11 \%$ respectively. Of the general practitioners, $39 \%$ did not know if there was one. In $96 \%$ of nursing homes and $56 \%$ of general practices there seem to be a treatment protocol available, it was lacking in $2 \%$ and $17 \%$ respectively, and $2 \%$ of nursing home physicians and $28 \%$ of general practitioners did not know.

None of the nursing home physicians, general practitioners or pharmacist shaves reported to be financially compensated for performing a medication review. All nursing home physicians, general practitioners and pharmacists stated to consider medication reviewing to be economically valuable and contributing to good quality of care.

\section{Discussion}

Performing proper medication reviews for multi-morbid older people is a challenging but essential process to prevent medicationrelated morbidity and adverse events. This study showed that there was substantial inconsistency in the frequency of reviewing, ranging from monthly (in 40\%) by the nursing home physicians to four times a year (in 50\%) by the general practitioners. In addition, the minimum requirement of reviewing every patient annually for communitydwelling patients or twice a year for nursing home residents was not met in daily practice.

Although there is consensus about the need to perform regular reviews, there is a lack of agreement about what a medication review should comprise. For example, there is no consensus on the best frequency in which a medication review should be performed, who the best professional is to carry out medication reviews and so on. Apart from this national discussion there is much heterogeneity between countries in and outside Europe in how medication reviewing should be managed. In the US for instance, pharmacists are obligated to review their nursing home patients once a month [14]. For community dwelling patients, a service model called Medication Therapy Management (MTM) is developed for older patients who meet certain criteria such as transition of care, prescription of five or more medications or a patient who has one or more chronic diseases. MTM consists of five elements, medication therapy review, personal medication record, medicationrelated action plan, intervention or referral, documentation and followup [15]. In Australia, the Department of Health and Aging published revised guiding principles for medication management in residential homes in 2012. Residential homes in Australia are obligated to have Medication Advisory Committees that monitors the medication management process. This committee comprises representatives of the management, nurses, medical practitioners and pharmacists and they will meet on a regular basis [16]. In the community, other Medication Management Review (MMR) programs are required such as the Home Medicine Review which involves the pharmacist visiting the individual at home, reviews their medicine regime and reports back to the general practitioner [17].

Despite our best efforts, still significant improvements could be made. In 2011 the British Geriatrics Society published a report in which they also reflected on medication management. They concluded that medication management in the UK care homes could be improved and they recommended to carry out regular medication reviews to reduce medication error, however without stating the frequency [6] In the Netherlands, the Dutch Health Care Inspectorate published a critical report in 2010 in which they concluded that improvement of medication safety was necessary in more than $75 \%$ of the investigated nursing homes and in almost $100 \%$ of the homes for the elderly [13] The main conclusions were that the physician and nursing staff did not always have an up to date medication overview, the professionals did not perform a medication review once (community) or twice (nursing home) a year. In the homes for the elderly the nursing staff often did not know whether the patient was capable to manage his own medication. Furthermore medication signalling, for example regarding interactions or dosage adjustment advices, was not handled in time and there was no clear case manager regarding pharmaceutical care [13].

Time spent on one review varied significantly between both groups, namely 10 minutes for a nursing home physician and 20 minutes for general practitioners. This difference in invested time might be explained by the fact that nursing home physicians care for different types of patients than the general practitioner. Also, this difference could be a reflection of the fact that medication reviews are performed differently by nursing home physicians and general practitioners. There was no significant correlation between the frequency of reviewing and time spent on one medication review. However, to the best of our knowledge there are no data on this and further investigation of this issue is needed.

The report of the Dutch Health Care Inspectorate also states that the reviews should be performed by the physician and pharmacist, nursing staff and/or patient [13]. In daily practice, meetings between the physician and pharmacist indeed took place regularly (91\%), but these were mainly organised as pharmacotherapy audit meetings and not for medication reviewing of individual patients.

In addition, this study showed that the medication data transmission between professionals could be improved. When medication was changed by another doctor, e.g. by a hospital specialist, only $47 \%$ of nursing home physicians and $44 \%$ of the general practitioners were informed often and even $13 \%$ of nursing home physicians and $6 \%$ of general practitioners never received any notice. Seventy-seven per cent of the physicians themselves always notified the pharmacy when stopping drugs and 5\% never did this. In daily practice, it is often difficult to establish who is primarily responsible for an up-to-date medication plan of an individual patient. Older people often have many drugs prescribed by different doctors. As a result, many physicians are involved in the treatment of one individual patient. This may lead to the feeling of not being responsible or incompetency regarding altering medications prescribed by a colleague [18]. In our opinion, the nursing home physician and general practitioner should be considered as the case-manager for their patients regarding pharmaceutical care.

With regard to what kind of information was used when reviewing, $88 \%$ used laboratory values and $75 \%$ used patient characteristics such as height and length. In daily practice, different professionals use different information sources in performing a review. For instance, a pharmacist only has access to the medication list, name and age of a 
patient whereas a general practitioner implements the medical history and laboratory results. Until now, there are no data about which data are important to perform a good review. Future research on this subject is needed to optimise the quality of medication reviews.

An alarming finding of our study was that $59 \%$ of the nursing home physicians and $89 \%$ of general practitioners considered workload to be an important limiting factor in performing reviews. This is an important finding which suggests that, however plausible, the recommendations made by the Dutch Health Care Inspectorate to perform a medication review for every patient once or twice a year, may not be achievable in the current situation [13]. Several studies in different settings have been performed in an attempt to guide, improve and assist the physician in performing medication reviews [19-21]. These studies have looked at educating professionals, development of computerised decision making systems, clinical pharmacy interventions, multidisciplinary approaches and combinations. These studies have shown mixed results, which leads to even more uncertainties about which review method is superior and achievable for daily practice.

The results of this survey imply that development of a novel and different medication surveillance system that is easy to use, does not add to, or even decreases the workload and can act on a continuous basis may help doctors in performing medication reviews.

This survey was limited in that the participants were only recruited in the south of the Netherlands and may therefore not be representative for other areas. Also the number of participants is small, especially the general practitioners group. Another limitation is that the results are self-reported and not objectively measured. Subsequently the interviewees may show a natural tendency to project a more positive image because they may be more committed in performing medication reviews.

To conclude, this survey shows great inconsistency in the way medication reviews are done. The results reflect how professionals define medication review themselves, which deviates on many aspects from the national recommendations. Collaboration with the pharmacist and other colleagues is thought to be important but requires improvement. Work load was said to be a major limitation in performing medication reviews which means that the minimum advices in the report of the Dutch Health Care Inspectorate in the current situation may not be attainable. This means that, to achieve a high standard, we may have to reconsider the way medication reviews are done.

\section{Acknowledgments}

This project was supported by a grant of the Netherlands Organisation for Health Research and Development (ZonMw) [40-41600-98-9009].

\section{References}

1. Olsson IN, Runnamo R, Engfeldt $P$ (2011) Medication quality and quality of life in the elderly, a cohort study. Health Qual Life Outcomes 9: 95.

2. Fortin M, Lapointe L, Hudon C, Vanasse A (2005) Multimorbidity is common to family practice: is it commonly researched? Can Fam Physician 51: 244-245.

3. Kaufman DW, Kelly JP, Rosenberg L, Anderson TE, Mitchell AA (2002) Recent patterns of medication use in the ambulatory adult population of the United States: the Slone survey. JAMA 287: 337-344.

4. Central Bureau voor Statistiek.
5. Barber ND, Alldred DP, Raynor DK, Dickinson R, Garfield S, et al. (2009) Care homes' use of medicines study: prevalence, causes and potential harm of medication errors in care homes for older people. Qual Saf Health Care 18: 341-346.

6. British Geriatrics Society (2011) Quest for quality; an inquiry into the quality of healthcare support for older people in care homes: a call for leadership, partnership and improvement.

7. Gurwitz JH, Field TS, Harrold LR, Rothschild J, Debellis K, et al. (2003) Incidence and preventability of adverse drug events among older persons in the ambulatory setting. JAMA 289: 1107-1116.

8. Bates DW, Cullen DJ, Laird N, Petersen LA, Small SD, et al. (1995) Incidence of adverse drug events and potential adverse drug events. Implications for prevention. ADE Prevention Study Group. JAMA 274: 29-34.

9. Juurlink DN, Mamdani M, Kopp A, Laupacis A, Redelmeier DA (2003) Drug drug interactions among elderly patients hospitalized for drug toxicity. JAMA 289: $1652-1658$

10. Leendertse AJ, Egberts AC, Stoker LJ, van den Bemt PM; HARM Study Group (2008) Frequency of and risk factors for preventable medication-related hospital admissions in the Netherlands. Arch Intern Med 168: 1890-1896.

11. Hanlon JT, Schmader KE, Ruby CM, Weinberger M (2001) Suboptima prescribing in older inpatients and outpatients. J Am Geriatr Soc 49: 200-209.

12. Schols JM (2005) Nursing home medicine in The Netherlands. Eur J Gen Pract 11: 141-143.

13. Ministery of Health (2010) Medicatieveiligheid voor kwetsbare groepen in de langdurige zorg en zorg thuis onvoldoende. Rapport Inspectie voor de Gezondheidszorg.

14. Furniss L, Burns A, Craig SK, Scobie S, Cooke J, et al. (2000) Effects of a pharmacist's medication review in nursing homes. Randomised controlled trial. Br J Psychiatry 176: 563-567.

15. American Pharmacists Association and the National Association of Chain Drug Stores Foundation ((2008) Core Elements of an MTM Service Mode Medication Therapy Management in Pharmacy Practice.

16. Australian Government: Guiding principles for medication management in residential aged care facilities (2012) Aging DoHa.

17. Australian Government (2013) Medication Management Review (MMR) Aging DoHa.

18. Hurkens KPGM, de Wit HAJM, Mestres Gonzalvo C, van der Kuy PHM Janknegt $\mathrm{R}$ et al. (2012) Medicatiemanagement bij de oudere patiënt: de ontwikkeling van SCREEN, Tijdschrift voor ouderengeneeskunde 123-127.

19. Bates DW, Gawande AA (2003) Improving safety with information technology N Engl J Med 348: 2526-2534.

20. Kaboli PJ, Hoth AB, McClimon BJ, Schnipper JL (2006) Clinical pharmacists and inpatient medical care: a systematic review. Arch Intern Med 166: 955-964.

21. Schnipper JL, Rothschild JM (2012) Improving medication safety. Lancet 379 1278-1280. 